\title{
Efeito do tratamento com acupuntura em éguas, para indução do cio
}

\section{Effect of the acupuncture treatment on the induction of estrous in mares}

\author{
Marcello Martinho Costa, ${ }^{*}$ André Vianna Martins, ${ }^{*}$ Tetsuo Inada, ${ }^{\star *}$ Marcio Ricardo costa dos Santos ${ }^{\star \star \star}$
}

\begin{abstract}
Resumo
Este trabalho tem como objetivo avaliar o efeito da acupuntura na indução de estro em nove éguas Puro-Sangue Inglês virgens, que tiveram retardo no aparecimento de estro na estação de monta, comparado a um grupo controle de 12 animais. Utilizaramse os pontos 23B (Shenshu) e Bai Hui, para aplicação de eletroacupuntura com freqüência de $10 \mathrm{~Hz}$, por 10 minutos, uma vez por semana, durante duas semanas. Observou-se o aparecimento de estro após a primeira sessão de acupuntura, obtendose um tempo médio de aparecimento, em dias, de 13,66 $\pm 9,21$. A duração do estro do grupo tratado apresentou uma média de $5,11 \pm 2,4$ dias, enquanto o grupo controle apresentou uma média de $7,2 \pm 2,91$ dias. A taxa de prenhez à primeira cobertura foi de $88,88 \%$ no grupo tratamento e de $58,33 \%$ no grupo controle. A taxa de prenhez, incluindo todas as coberturas, permaneceu igual no grupo tratado e subiu para $83,33 \%$ no grupo controle. Dos resultados obtidos pode-se concluir que a acupuntura é uma alternativa para auxiliar o tratamento de éguas que não apresentam ciclo estral no início da estação de monta.
\end{abstract}

Palavras-chave: acupuntura; estro; égua.

\begin{abstract}
This work has how objectivo to valuated the effect of acupuncture treatment in induction of estrus in 09 Thorough-bred, virgins mares that showed delaying of estrus expression in the breeding, when compared to a control group of 12 animals. It was used the 23B ( Shenshu ) and Bai Hui points for the application of electroacupuncture over 10 minutes, with $10 \mathrm{~Hz}$ frequency, one a week, during two weeks. It was observed the estrus expression, after the first treatment with acupuncture, obtaining a mean time of estrus detection of $13,66 \pm 9,21$ days. The duration of estrus from treated group showed a mean time of $5,11 \pm 2,4$ days, while the control group showed a mean time of $7,2 \pm 2,91$ days. The pregnancy level at first mount was $88,88 \%$ for the treated group and $58,33 \%$ for the control ones. The pregnancy level, including all the mounts, was $88,88 \%$ for the treated group and $83,33 \%$ for the control group. From the results obtained, it can be concluded that acupuncture is an alternative to help the treatment of mares that do not show estrous cycle in the beginnig of breeding season.
\end{abstract}

Keywords: acupuncture; estrus; mare.

\section{Introdução}

Os primeiros relatos da acupuntura veterinária na China datam do século XI a.C. no escrito Li-Ji, em que cavalos selecionados para as batalhas eram preparados através da acupuntura. Ao longo das dinastias chinesas, vários escritos foram surgindo tanto da acupuntura humana quanto da acupuntura veterinária, sendo o mais importante deles, para ambos, o Hung-di Ne-Jing (The Yellow Emporo's Classic of Internal Medicine) escrito no século III a.C. pelo imperador Huang Di. No século XX, em 1947, o Agricultural College of The Northern University incorporou o estudo da acupuntura veterinária em seu currículo. Com isso, o estudo da Medicina Tradicional Chinesa ganhou impulso a partir da fundação da República Popular da China, em 1949.
O conceito Yin e Yang é um dos aspectos do materialismo simples e da dialética da filosofia chinesa taoísta, utilizado pela Medicina Tradicional Chinesa para explicar as funções fisiológicas do organismo, as alterações patológicas e as relações internas dos órgãos, assim como sua aplicação no diagnóstico e no tratamento (Mann, 1982 ; Sumano, López, 1990 ; Wenbu, 1993).

O Yin e Yang são duas energias opostas e que estão em constante processo de transformação e transmutação. Estas energias são a base da vida do universo. Os fenômenos da natureza, os organismos vivos, todos os elementos da natureza possuem características Yin e Yang. O Yin é representado pelo frio, noite, obscuro, interior, feminino, imobilidade, água, preto, negativo, passivo; o Yang é representado pelo

\footnotetext{
* Médico Veterinário Autônomo.

** Prof. Adjunto IV - Universidade Federal Rural do Rio de Janeiro.

${ }_{\star \star \star *}$ Prof. Adjunto - Universidade Federal Fluminense.
} 
calor, dia, claro, exterior, masculino, mobilidade, fogo, branco, positivo e o ativo (Klide, 1977 ; Mann, 1982 ; Sumano, López, 1990 ; Wenbu, 1993).

Um indivíduo é considerado saudável quando as duas energias estão em equilíbrio. Quando ocorre o desequilíbrio entre Yin e Yang, manifesta-se a doença. $O$ desequilíbrio entre Yin e Yang pode ser por excesso de Yang ou de Yin ou por deficiências de Yin ou Yang. O excesso é conhecido como "Síndrome Shi" e a deficiência é conhecida como "Síndrome Xu", na Medicina Tradicional Chinesa (Klide, 1977 ; Mann, 1982 ; Sumano, López, 1990 ; Wenbu, 1993).

É a partir das características das Síndromes Yin e Yang que é realizado o diagnóstico e o princípio para o tratamento em que seda-se o excesso e tonifica-se a deficiência para restabelecer o equilíbrio entre as duas energias (Klide, 1977 ; Mann, 1982 ; Sumano, López, 1990 ; Wenbu, 1993).

\section{Material e métodos}

Foram utilizadas nove éguas virgens da raça Puro-Sangue Inglês, alojadas no Posto de Fomento Antônio Carlos Amorin, de propriedade da Associação de Criadores e Proprietários de Cavalo de Corrida do Rio de Janeiro (ACPCC-RJ), localizado no Km 71,5 da Rio-Bahia, Três Córregos, Teresópolis $R J$, lat $=-22^{\circ} 20^{\prime} 38^{\prime \prime}$ e long $=-42^{\circ} 56^{\prime} 22^{\prime \prime}$. O experimento foi realizado no período de 15 de agosto/93 a 15 de janeiro de 1994, que compreende a estação de monta. O período de luz diário medido todo dia 15 de cada mês, no ano de 1994, apresentou um tempo médio entre os meses de agosto e dezembro de $12 \mathrm{~h} 52 \pm 0,8 \mathrm{~h}$, tendo o dia 15 de agosto um período de luz de $11 \mathrm{~h} 19$ e no dia 15 de dezembro, um período de $13 \mathrm{~h} 32$, tempo este medido na cidade do Rio de Janeiro (Brasil, 1994). As reprodutoras, antes de entrarem para a estação de monta, foram submetidas a um exame clínico geral e reprodutivo, e rufiadas diariamente para a detecção do cio. Detectado o cio, foram feitos exames ultra-sonográficos e através da palpação retal, para avaliar o desenvolvimento folicular. Esta avaliação tem por objetivo determinar o melhor momento para a cobertura. É utilizado o regime de monta natural. Algumas éguas virgens, que apresentam hipofunção ovariana, são submetidas ao tratamento por fotoperíodo, com luz artificial de 250 watts na cocheira, para totalizar um período de luz diário de 16 horas. Os animais recebem uma alimentação à base de aveia, alfafa, ração concentrada, forragem picada, cenoura, sal mineral e suplementação vitamínica.

As éguas foram selecionadas segundo os critérios de apresentarem um desenvolvimento folicular insatisfatório e em decorrência disto, não apresentavam estro. O tempo médio de permanência no Posto de Fomento sem a manifestação de estro, no início do tratamento com acupuntura foi de 30 dias. As éguas foram submetidas a exames clínicos, não sendo constatadas anormalidades que justificassem o não aparecimento do estro. Para o tratamento foram utilizados os pontos $23 \mathrm{~B}$
(Shenshu) e Bai Hui. A localização desses pontos é, respectivamente, 23B, a 1,5 Tsun lateral à linha média dorsal entre o processo espinhoso das vértebras lombares L2 e L3. Bai Hui, na linha média dorsal, na depressão entre a última vértebra lombar e a primeira vértebra sacral (Figura 1). O Tsun é uma medida utilizada pela Medicina Tradicional Chinesa para localização dos pontos acupunturais, e 1 Tsun corresponde à largura de uma costela. Foram utilizadas agulhas de aço inoxidável de fabricação chinesa, próprias para eqüinos. 
O grupo controle constou de 12 animais, sendo que cinco receberam tratamento de luz artificial. Neste grupo foram avaliadas a taxa de prenhez à primeira cobertura, a taxa de prenhez total e a duração do cio.

Para análise estatística dos resultados utilizou-se o programa MINITAB (Schafer, Anderson, 1989).

\section{Resultados}

O aparecimento do cio variou de 2 a 27 dias após o tratamento. Obteve-se uma média de 13,22 $\pm 9,21$ dias. O tempo de duração do cio variou de 2 a 10 dias, com tempo médio de $5,11 \pm 2,4$ dias. A taxa de prenhez ao primeiro cio foi de $88,88 \%$. A taxa de prenhez total foi de $88,88 \%$. Um animal apresentou reabsorção embrionária por duas vezes, terminando a estação com prenhez negativa, como pode ser observado nas Tabelas 1 e 2.

Tabela 1: Duração de cio e resultados da prenhez à $1^{\text {a }}$ cobertura em éguas tratadas com acupuntura e as mantidas como controle

\begin{tabular}{|c|c|c|c|c|}
\hline \multirow[b]{2}{*}{ Animal } & \multicolumn{2}{|c|}{ GRUPO TIAT AMENTO } & \multicolumn{2}{|c|}{ GRUPO CONTROLE } \\
\hline & $\begin{array}{c}\text { Duração de } \\
\text { cio (a) }\end{array}$ & $\begin{array}{c}\text { Prenhez à } \\
1 \text { cobertura }(c)\end{array}$ & $\begin{array}{c}\text { Duração } \\
\text { de cio }\end{array}$ & $\begin{array}{l}\text { Prenhez à } \\
1^{\mathrm{a}} \text { cobertura (d) }\end{array}$ \\
\hline 1 & 7 & pos. & 15 & pos. \\
\hline 2 & 10 & pos. & 4 & pos. \\
\hline 3 & 7 & pos. & $9,5^{\star}$ & neg. \\
\hline 4 & 5 & pos. & $7,5^{\star}$ & neg. \\
\hline 5 & 2 & pos. & $5^{\star}$ & neg. \\
\hline 6 & 2 & pos. & 7 & pos. \\
\hline 7 & 4 & pos. & 7 & pos. \\
\hline 8 & 5 & pos. & 10 & pos. \\
\hline 9 & $4,5^{*}$ & neg. & 5 & pos. \\
\hline 10 & - & - & 5 & pos. \\
\hline 11 & - & - & $5.5^{\star}$ & neg. \\
\hline 12 & - & - & $7^{\star}$ & neg. \\
\hline
\end{tabular}

$\mathrm{P} \leq 0,01 \propto=0,01$, a: Duração de cio em dias, $\mathrm{b}:{ }^{*}$ Valor médio em dias de animais, que apresentaram mais de um cio, $c: p \leq 0,01$, d: $p \leq 0,01$.

Tabela 2: Tempo de aparecimento do cio em éguas após tratamento com acupuntura e em mantidas como controle

\begin{tabular}{|c|c|c|c|c|}
\hline \multirow[b]{2}{*}{ Animal } & \multicolumn{2}{|c|}{ GRUPOTRAT AMENTO } & \multicolumn{2}{|c|}{ GRUPOCONTROLE } \\
\hline & $\begin{array}{l}\text { Início de cio } \\
\text { (a) Pós- } \\
\text { tratamento }\end{array}$ & Prenhez (c) & $\begin{array}{l}\text { Início de ció } \\
\text { pós-tratamento }\end{array}$ & Prenhez (d) \\
\hline 1 & 3 & pos. & - & pos. \\
\hline 2 & 3 & pos. & - & pos. \\
\hline 3 & 27 & pos. & - & pos. \\
\hline 4 & 24 & pos. & - & pos. \\
\hline 5 & $18^{\star \star}$ & pos. & - & pos. \\
\hline 6 & $11^{\star \star}$ & pos. & - & pos. \\
\hline 7 & 23 & pos. & - & pos. \\
\hline 8 & 12 & pos. & - & pos. \\
\hline 9 & 2 & neg. & - & pos. \\
\hline 11 & - & - & - & neg. \\
\hline 12 & - & - & - & neg. \\
\hline
\end{tabular}

a: $p \leq 0,01, b:{ }^{\star \star}$ Cio silencioso, $c: p \leq 0,01, d: p \leq 0,05$.
No grupo controle observou-se uma taxa de prenhez à $1^{\text {a }}$ cobertura de $58,33 \%$ e uma taxa de prenhez total de $83,33 \%$ (Figura 2). O tempo médio de duração de cio foi de 7,2 $\pm 2,91$ dias com uma variação de 4 a 15 dias. Um animal apresentou reabsorção embrionária após a 1ª cobertura, apresentando prenhez na $2^{\mathrm{a}}$ cobertura. Dois animais apresentaram prenhez negativa (Tabelas 1 e 2 ). O grupo tratado e o grupo controle foram diferentes ( $p \leq 0,01$ ) em relação à taxa de prenhez à $1^{\text {a }}$ cobertura.



Figura 2: Taxa de prenhez à $1^{\text {a }}$ cobertura e prenhez final em éguas tratadas com acupuntura (grupo 1) e as controle (grupo 2)

\section{Discussão}

Dos poucos trabalhos científicos sobre acupuntura na espécie eqüina, somente um refere indicações de acupontos para o tratameto de problemas reprodutivos (Jingchang, 1987), onde utiliza o ponto YinTi.

O acuponto 23B ou Shenshu foi escolhido como um dos pontos para o tratamento, pois é o ponto de assentimento do órgão rins (Mann, 1982, Bastos, 1993). Os rins são órgãos que, dentre as suas funções, dominam o aparelho reprodutor (Wenbu, 1993).

O acuponto Bai Hui (ponto extra) e o ponto $23 \mathrm{~B}$, juntamente com outros pontos, são indicados para tratamento de problemas reprodutivos (Korematsu et al., 1993 ; Zhongjie et al., 1993 ; Lin, Panze, 1992 ; López, Buéndia, 1990 ; Hernandez et al., 1989 ; Gilchris, 1988 ; Pineda et al., 1987 ; Vazquez et al., 1987 ; Briñas, 1986 ; Huang, Dobson, Fitzpatrick, 1986 ; Malven, 1983).

A associação dos acupontos 23B e Bai Hui foi escolhida com o intuito de observar a resposta ao tratamento com apenas dois acupontos, no sentido de obter uma resposta satisfatória do tratamento com a diminuição de acupontos, sugestão também proposta por Wembu (1995) na escolha dos acupontos mais eficazes.

Neste trabalho foi possivel observar uma discordância com relação à nomenclatura e à localização de alguns acupontos, entre a literatura da acupuntura humana e a da acupuntura veterinária. $\mathrm{O}$ acuponto $23 \mathrm{~B}$, na literatura humana, é denominado Shenshu, e é o ponto de assentimento dos rins (Wenbu, 1993).

$\mathrm{Na}$ literatura veterinária, o acuponto 23B é denominado de Pangguangshu, e é colocado como ponto de assentimento da bexiga. Esta denominação é feita tanto por chineses como 
por ocidentais Klide (1977). Porém Klide (1977), em seu livro, colocou uma interrogação nos acupontos 23B e 27B, referindo-se qual deles é realmente o ponto de assentimento dos rins. Para Wenbu (1993), Pangguangshu corresponde ao acuponto 28B. No presente trabalho observa-se que apenas um autor veterinário cita 0 23B como sendo o Shenshu, porém sem fazer referência se este é o ponto de assentimento dos rins (Gilchiris, 1988). Traduzindo: Shen significa rins, Shu assentimento; logo, Shenshu significa assentimento dos rins. Pangguang significa bexiga, logo, Pangguangshu é o ponto de assentimento da bexiga.

Desta maneira, optou-se neste trabalho por usar a indicação do acuponto $23 \mathrm{~B}$, descrito tanto na literatura humana, quanto no trabalho de Gilchris (1988).

$\mathrm{O}$ acuponto Bai-Hui é considerado um ponto extra. Na literatura veterinária localiza-se medialmente no espaço entre a última vértebra lombar e a $1^{\text {a }}$ sacral (Klide (1977) e demais autores veterinários). Porém, na literatura acu-humana, o ponto Bai Hui localiza-se no ponto 20VG (Wenbu, 1993); assim sendo, este ponto foi escolhido como o indicado na literatura veterinária.

A eletroacupuntura vem sendo utilizada por apresentar, entre outras vantagens, redução no tempo de tratamento em relação à acupuntura clássica (Bastos, 1993). Estipulou-se o tratamento uma vez por semana, por duas vezes, tratamento que se assemelha ao preconizado por Gilchris (1988), para distúrbios ovarianos. O tempo de duração do tratamento foi estipulado em 10 minutos, o mesmo preconizado pela literatura quando a intenção é tonificar o órgão. Bastos (1993) afirma que, na tonificação com a eletroacupuntura, o tratamento deve ter um tempo de duração de até 15 minutos, com uma freqüência de 5 a $10 \mathrm{~Hz}$.

As éguas virgens, submetidas ao tratamento, apresentavam uma hipofunção ovariana. Não apresentavam estro. Dentro da Medicina Tradicional Chinesa, uma hipofunção é vista como uma patologia de características Yin (Klide, 1977 ; Fernández, 1977 ; Jaggar, 1992 ; Wenbu, 1993 ; Bastos, 1994). Há um desequilíbrio entre Yin e Yang, onde o Yin está em excesso em relação ao Yang. Porém este desequilíbrio é causado pela deficiência de Yang, síndrome do frio do tipo $\mathrm{Xu}$, levando a manifestações de características Yin. Os rins possuem, entre outras funções, o controle dos órgãos reprodutores. Um desequilíbrio no órgão rins leva a distúrbios reprodutivos, que podem manifestar-se de diversas formas. Wenbu (1993) afirma que uma deficiência de Yang, levando à preponderância de Yin, pode causar infertilidade. Klide (1997) afirma que o tratamento dos processos reprodutivos em fêmeas deve ser feito com o fortalecimento dos rins; portanto, o tratamento destes animais visou ä tonificação do Yang dos rins.

Os animais tratados foram provenientes do Jockey Clube Brasileiro. São animais que estavam em regime de corridas, submetidos a um regime de esforço físico intenso, a um regime de estresse. De acordo com a Medicina Tradicional Chinesa, o aparecimento da doença ocorre quando há um desequilíbrio energético. Wenbu (1993) considera o esforço físico intenso como um dos fatores que podem levar a um desequilíbrio energético. Este desequilibrio estaria ocorrendo pelo consumo excessivo do Yang. Porém, considerar apenas um fator como responsável pelo desequilíbrio, seria fugir do pensamento da Medicina Tradicional Chinesa: para ela , o indivíduo faz parte de um todo, o universo (Mann, 1982). Este indivíduo deve estar em perfeito equilíbrio com a natureza. Quando se retira um animal de seu habitat natural, e o submete a trabalhos intensos, há vários fatores responsáveis pelo desequilíbrio energético deste animal.

A mudança de local, do Jockey Clube Brasileiro para o Posto de Fomento Antônio Carlos Amorin, é outro fator a ser considerado. Existe uma variação de adaptabilidade, observável pelo comportamento, de animal para animal. Animais que se adaptam com facilidade ao manejo do posto provavelmente apresentarão manifestação de estro mais rápido. Em eqüinos, a própria mudança de um tratador é um fator importante que influi na adaptação do animal. O contato com outros animais também tem sido um fator importante, pois eqüinos têm a característica de formar grupos sociais.

Das éguas do grupo tratamento, o aparecimento de cio após a $1^{\text {a }}$ sessão de acupuntura variou de dois a 24 dias, com uma média de 13,66 \pm 9,21 dias. Lin, Panzer (1992) afirmaram que o aparecimento de cio após $0.1 \%$ tratamento com acupuntura foi de três a 10 dias. Do grupo tratamento, seis animais apresentaram cio após 10 dias. Andrade (1983) afirma que a estação de monta no sudeste compreende do mês de outubro a janeiro. Dentro deste período, tem-se a atividade ovariana máxima. Mckinnon, Voss (1993) referiram que na égua existe um período de transição entre o final do inverno e o início da primavera, para o aparecimento de cio. Este período varia de seis a oito semanas ou até mais, podendo apresentar um desenvolvimento folicular irregular que dure meses. Diante destas afirmações, pode-se especular que, na avaliação de resposta ao tratamento, deve-se levar em consideração as características individuais com relação a este periodo de transição. A acupuntura age equilibrando o organismo. Uma vez equilibrado, o organismo irá apresentar uma resposta orgânica, no caso, o cio; de acordo com a natureza do animal. Andrade (1983) afirmou que o ciclo estral varia de 21 a 22 dias com extremos normais de 16 a 28 dias, sendo possível, assim, que a variação do tempo da manifestação do cio também esteja relacionada com a fase do ciclo estral em que foi iniciado o tratamento. Em animais quando o tratamento foi iniciado próximo ao período estral, onde o desequilíbrio energético não era grande, se observa uma resposta mais rápida. Em animais cujo tratamento iniciou-se logo após o término do período de estro, a resposta tem sido mais demorada, mas virá no próximo estro, daí o tempo encontrado de até 27 dias.

A taxa de prenhez ao $1^{\circ}$ cio, no grupo controle, foi de $58,33 \%$. No grupo tratamento esta taxa ficou em $88,88 \%$. Apesar do pequeno número de animais utilizados neste trabalho, foi possivel observar diferença significativa $(p \leq 0,01)$ quando tratados por acupuntura. Outro fator importante a ser observado foi que os animais do grupo controle eram animais sadios, pois apresentaram estro dentro de um período de no máximo 15 dias a partir da entrada no Posto de Fomento, não havendo necessidade de tratamento. Os animais do grupo foram selecionados entre os que não apresentavam estro. Esta diferença sugeriu uma eficácia da acupuntura na indução de estro $(p \leq 0,01)$ com ovulação. Mckinnon (1993) afirmou que da observação do $1^{\circ}$ desenvolvimento folicular até a $1^{\text {a }}$ ovulação, podemos ter um período de 55 a 60 dias. Este período compreende a transição do final do inverno ao início da pri- 
mavera. Afirmou ainda este autor, que deve-se levar em consideração a qualidade do oócito e as condições do útero para haver a fecundação. No período de transição, algumas fêmeas podem desenvolver de 3 a 4 folículos anovulatórios antes da primeira ovulação. No mesmo ano, Morrow afirmou que, no período de transição, tem ocorrido o desenvolvimento folicular pela ação do FSH, mas não se tem a ovulação, pois - LH encontra-se em níveis basais. Os resultados obtidos sugerem que a acupuntura pode viabilizar este primeiro cio, induzindo a uma ovulação.

\section{Referências bibliográficas}

ANDRADE, L. S. Fisiologia e manejo da reprodução eqüina. Recife : Parque Gráfico da Fábrica de Discos Rozemblit, 1983. $388 p$.

ANTONIO, F.F. Tratado general de acupuntura china : fisiopatologia, diagnóstico y tratamiento. Buenos Aires : Ediestrones CientíficoTécnicos Americanas, 1977. 427 p.

BASTOS, R. S. R. C. Tratado de eletroacupuntura : perspectivas científicas, teoria e prática. Rio de Janeiro: Numen Editora, 1993. $492 \mathrm{p}$.

BRASIL - MINISTÉRIO DA CIÊNCIAE TECNOLOGIA. Efemérides astronômicas. Rio de Janeiro: Polar, 1994. 325 p.

BRIÑAS, P. Sobre el empleo de la acupuntura en algunos transtornos de la reproducestron en hembras bovinas. ENCUENTRO NAESTRONAL DE LA SOCIEDAD CUBANA DE ACUPUNTURA EN LA MEDICINA VETERINARIA, 1., Anais... Cuba : Centro de Posgrado del IMV. $39 p$.

CHENGMIN, L. et al. Investigations on Lasemuncture For The Treatament of Ovarian and Uterus Diseases and Conception in The Dairy Cattle. INTERNATIONAL CONFERENCE ON VEIERINARY ACUPUNCTURE, 1., 1987, Beijing. Abstracts... Beijing. China Academic Publishers, 1987. p. 70-71.

DEEN, L., G. Z., ZHENBANG, R. Efect Of The Laser Acupuncture on Acyetic Treatment and Fetus Protection in Cows. INTERNAL CONFERENCE ON VETERINARY ACUPUNCTURE, 1., 1987, Beijing. Abstracts... Beijing. China Academic Publishers, 1987. p. 74.

GILCHRIST, D. Equine Acupuncture. Kuranda:Pro-Prom PTY, 1988. $96 \mathrm{p}$.

HERNANDEZ, J.M. et al .Effects of Acupuncture on The Treatment of Repeat Breeding Zebu Cattle. American Journal of Acupuncture, v. 17, n. 4, p. 337-341, 1989.
A taxa de prenhez total foi semelhante para os dois grupos, sendo que os dois animais do grupo controle que ficaram vaziós não apresentaram ovulação. Por outro lado, a égua vazia do grupo tratamento apresentou ovulação.

\section{Conclusões}

De acordo com os resultados obtidos, pode-se concluir que o tratamento das éguas PSI pela acupuntura utilizando os acupontos 23B (Senshu) e Bai Hui foi eficaz na indução do estro.

HUANG, Y.D., DOBSON, H., FITZPATRICK, R.J. Plasma Hormone Response After Acupuncture Treatment of Bovine Subfertility. Animal Reproduction Science, Amsterdam, v. 11. n. 3, p. 173-180, 1986.

KLIDE, A.M., KUNG, S.H. Veterinary Acupuncture. Pennsylvania : University of Pennsylvania Press, 1977.297 p.

KOREMATSU, Ki., et al. Therapeutic Effects of Moxibustion on Delayed Uterine Involution in Pospartum Dairy Cows. Journal Veterinary Medicine Science, v. 55, n. 4, p. 613-616, 1993.

McKINNON, A. O. VOSS, J. L. Equine Reproduction. Malvern. Lea \& Febiger, 1994.1137p.

MANN, F.. Acupuntura. A arte chinesa de curar. São Paulo : Hemus, 1982. $208 \mathrm{p}$.

MALVEN, P.V. BOSSUT, D.f.b. DIEKMAN, M.A. Effects of Naloxone and Eletroacupunture Treatment on Plasma Concentrations of LH in Sheep. Journal of Endocrinology, London, v.101, n. 1, p. 75-80. 1984.

MORROW, D. A. Diagnosis Treatment and Prevention of Reproductive Diseases in Small and Large Animals. Philadelphia. W. B. Saunders Company, 1986. 1143 p.

PINEDA, F.J.Z., BERMUDEZ, J.P.R., PÉREZ, M.G.R. Evaluation of Acupuncture Treatment and Antibiotherapy in Chronic Endometritis if Holstein Friesian Cows. INTERNATIONAL CONFERENCE ON VETERINARY ACUPUNCTURE, 1., 1987, Beijing. Abstracts... Beijing. China Academic Publishers, 1987. p. 59-60.

SUMANO, L.M., LÓPEZ, B.G. Acupuntura Veterinaria. México : Interamericana, $1990.346 \mathrm{p}$.

VASQUEZ, F. Efecto de la acupuntura sobre la fertilidad de vacas repetidoras holstein. Revista Cubana de la Medicina Veterinaria, Cuba, v. 18, n. 3-4, p. 105-112, 1986.

ZHONGJIE, L. et al. Equine Acupuncture. Beijing : Beijing University Press, 1993.207 p.

WENBU, X. Tratado de medicina interna chinesa. São Paulo : Roca, 1993. 691 p. (Traduzido por Issao Yamamura). 